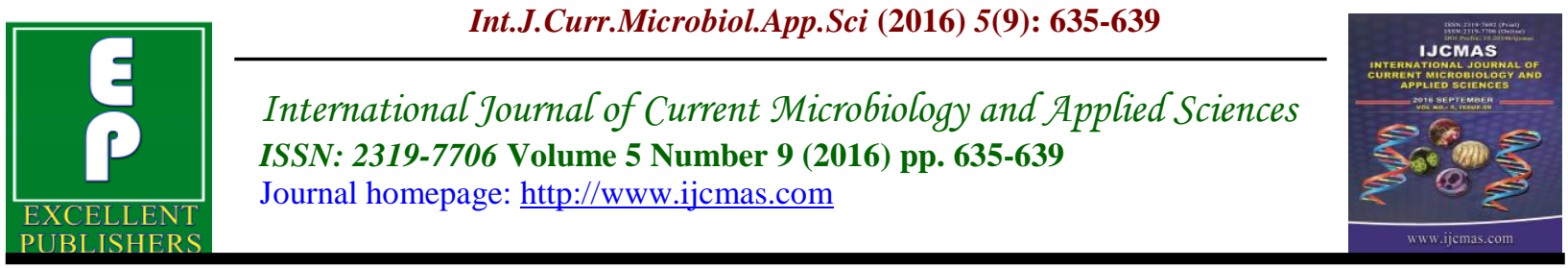

Original Research Article

http://dx.doi.org/10.20546/ijcmas.2016.509.072

\title{
A Study on Antibacterial Resistance Patterns on the Enterococcus Species Isolated in a Tertiary Care Hospital in South India
}

\author{
V. Dillirani* and R. Suresh \\ Department of Microbiology, Chengalpattu Medical College, Chengalpattu 603 001, India \\ *Corresponding author
}

Keywords

Enterococci, nosocomial infections, Antibiotic susceptibility, Vancomycin.

Article Info

Accepted:

25 August 2016

Available Online:

10 September 2016

\section{A B S T R A C T}

Enterococci are normal inhabitants of human colon and biliary tracts. In recent years, emerged as much important opportunistic pathogen of human diseases, causing life threatening hospital acquired infections. They have gained great significance globally, because of their increased resistance to the widely used antimicrobials and Vancomycin. The objective was to analyze antibiotic resistance pattern of enterococci from clinical samples. The current study was carried out in the department of Microbiology, Chengalpattu medical college from January 2015to December 2015. Enterococci isolated from the clinical samples like urine, blood pus and samples were confirmed and antibiotic susceptibility pattern were observed. Enterococcus spp. isolated in the present study from various clinical specimens was 120. Enterococcus faecalis (93.3\%) and Enterococcus faecium $(6.7 \%)$ were exclusively isolated species. Antibiotic susceptibility pattern revealed maximum resistant to Penicillin (91\%), followed by Ciprofloxacin(81\%), Ampicillin (69\%), Doxycycline (62\%) and high level Gentamycin (48\%). Among the isolates 3\% were resistant to Vancomycin. The rational and appropriate usage of antimicrobials in the community and health care centers will minimize the emerging multidrug and Vancomycin resistant strains of enterococci. Any therapeutic management should be based on the antibiotic policy of the concerned center and susceptibility pattern of the isolates from the clinical specimens.

\section{Introduction}

Enterococci are normal inhabitants of human gastrointestinal and biliary tracts, female genital tract and male urethra. They have emerged as important hospital associated pathogen causing both complicated and uncomplicated urinary tract infections, intravascular catheter associated blood stream infections, intra-abdominal infections, surgical site skin and soft tissue infections. Based on the Centers for Disease Control and Prevention's (CDC) data the enterococcal species are the second most 
common organism causing nosocomial infections worldwide (Desai et al., 2001).

Urinary tract infections are the most frequent enterococcal infection, mostly health care associated, secondary to catheterization or instrumentation, or due to structural abnormalities of the urinary tract (Schaberg et al., 1991). Enterococcal bacteremias are most often nosocomial in origin, associated with intravascular lines, urethral catheterization, long-term hospital stay and other sites infections. Enterococci are identified in 5-20\% of all cases of infective endocarditis and also in prosthetic valve endocarditis (Megam, 1991). They are the third most common pathogen isolated from wound infections.

Two species of Enterococci are the frequently isolated agents in human infections- Enterococcus faecalis in $80-90 \%$ and Enterococcus faecium in 5-10\% of enterococcal infections (Ross, 2006). The inherent resistance of Enterococci to commonly used antimicrobials agents and acquired resistance to aminoglycosides (high level resistance), glycopeptides, $\beta$ - lactams and fluroquinolones has increased their clinical importance in recent years. So the present study was conducted to know the antimicrobial resistance pattern and prevalence of Enterococci isolates from clinical samples.

\section{Materials and Methods}

The present study was a retrospective study conducted in a tertiary care centre in South India for a period of one year from January 2015 to December 2015. Aseptically collected clinical samples like urine, blood, pus and body fluids were processed by standard methods.

Enterococcus spp. isolated were confirmed by colony morphology, Gram's staining, catalase negativity, salt tolerance testgrowth in the presence of $6.5 \% \mathrm{NaCl}$, esculin hydrolysis in the presence of $40 \%$ bile salts, growth at $10^{\circ} \mathrm{C} \& 45^{\circ} \mathrm{C}$ and ability to produce PYR (Forbes et al., 2007; Collee et al., 2008). Enterococcus species was identified by using conventional Facklam and Collins biochemical tests based on fermentation of sugars, pyruvate utilization and arginine decarboxylation.

The antimicrobial susceptibility test by Kirby Bauer disc diffusion method was carried on 5\% Mueller Hinton blood agar, as per CLSI guidelines. All isolates were tested for following antimicrobials - Penicillin $(10 \mu \mathrm{g})$, Ampicillin $(10 \mu \mathrm{g})$, Doxycycline $(30 \mu \mathrm{g})$, Ciprofloxacin $(5 \mu \mathrm{g})$, Gentamycin $(200 \mu \mathrm{g})$. Vancomycin screen agar was used for the identification of Vancomycin resistant enterococci as recommended by CLSI.

\section{Results and Discussion}

Enterococcus spp. isolated in the present study from various clinical specimens was 120. Of the 120 isolates Enterococcus faecalis were 112 (93.3\%) and Enterococcus faecium were $8(6.7 \%)$.

The highest number of isolates were uropathogen 89 (74\%). Organisms isolated from pus, blood and tissue fluids were $18(15 \%), 7(6 \%)$ and $6(5 \%)$ respectively. Antibiotic susceptibility pattern revealed maximum resistant to Penicillin (91\%), followed by Ciprofloxacin(81\%), Ampicillin (69\%), Doxycycline (62\%) and high level Gentamycin (48\%). Enterococci were highly susceptible to Vancomycin (97\%). All Vancomycin resistant enterococci4(3.3\%) were isolated from urine specimen and all belong to Enterococus faecium species. Nearly $85 \%$ of the strains were isolated from hospitalized patients and $15 \%$ were from outpatient department. 
Table.1 Enterococcus species isolated from various clinical samples.

\begin{tabular}{|l|c|c|}
\hline Sample & Number of Isolates (120) & Percentage (\%) \\
\hline Urine & 89 & $74 \%$ \\
\hline Pus & 18 & $15 \%$ \\
\hline Blood & 7 & $6 \%$ \\
\hline Tissue Fluids & 6 & $5 \%$ \\
\hline
\end{tabular}

Table.2 Antibiotic resistance pattern of Enterococcus spp. isolated from various clinical samples.

\begin{tabular}{|l|c|c|}
\hline Antibiotics & Number of resistant Isolates & Percentage \\
\hline Penicillin & 109 & $91 \%$ \\
\hline Ampicillin & 83 & $69 \%$ \\
\hline Ciprofloxacin & 97 & $81 \%$ \\
\hline Doxycycline & 74 & $62 \%$ \\
\hline High Level Gentamycin & 57 & $48 \%$ \\
\hline Vancomycin & 4 & $03 \%$ \\
\hline
\end{tabular}

Enterococcus spp. were considered as low virulent resident flora of man, but very recently emerged as much important hospital acquired opportunistic pathogen of human diseases. They have gained great significance globally, because of their increased resistance to the widely used antibacterial drugs and transmission in hospitalized patients by contaminated hands of health care providers. The emergence of Vancomycin resistance in these bacteria is often involved in serious super infections among patients receiving broad spectrum antimicrobials therapy.

In this study $2.8 \%$ of organisms isolated from various clinical samples were Enterococci spp., almost the same results were obtained in the study conducted by Mukherjee et al. In our study $56 \%$ female were affected where as only $44 \%$ of male were infected with enterococcal infections, which was concurrence with study of Bose et al., (2012).

$\begin{array}{lllr}\text { Enterococcus } & \text { faecalis } & (93.3 \%) & \text { and } \\ \text { Enterococcus } & \text { faecium } & (6.7 \%) & \text { were }\end{array}$

exclusively isolated species, which was accordance with the findings of previous studies (Gullberg et al., 1989).

Enterococci isolated were predominantly from urine $(74 \%)$ specimen followed by pus $(15 \%)$ and blood $(6 \%)$, the results were similar with findings of Chakraborty et al., (2015). Regarding the antimicrobial susceptibility pattern of the isolates, they were more resistant to Penicillin (91\%), Ciprofloxacin (81\%) and Ampicillin (69\%). The results were almost in concurrence with some recent Indian studies (Parameswarappa et al., 2013). High level Gentamycin resistance in our study was $48 \%$, this observation was comparable with many studies (Oberoi et al., 2013; Fernandes et al., 2013).

In developing countries higher irrational empirical usage of antimicrobials resulted in increased therapeutic resistance of antibacterials in the community as compared to the developed nations, which is a real major concern in health system worldwide 
(Kumar et al., 2013). The high prevalence of Vancomycin resistant enterococci in the nosocomial infections was reported in many studies from all over the world, but in our study it was only 3\%. Near similar result was observed by Mukherjee et al.

In conclusion, in the present study the enterococci from various clinical samples were resistant to most of the commonly used antibacterials. Increased incidence of Vancomycin resistant enteroccocci from different studies emphasize the immediate need for intervention in current antimicrobial management and monitoring. Our study concludes that the rational and appropriate usage of antimicrobials in the community and health care centers will minimize the emerging multidrug and Vancomycin resistant strains of enterococci. Any therapeutic management should be based on the antibiotic policy of the concerned center and susceptibility pattern of the isolates from the clinical specimens.

\section{References}

Bose, S., Ghosh,, A., Barapatre, R. 2012. Prevalence of Drug Resistance among Enterococcus Species Isolated from A Tertiary Care Hospital. Int. J. Med. Health Sci., 1: 38-44.

Chakraborty, A., Pal, N.K., Sarkar, S., Gupta, M.S. 2015. Antibiotic resistance pattern of Enterococci isolates from nosocomial infections in a tertiary care hospital in Eastern India. J. Nat. Sci. Biol. Med., 6(2): 394-397.

Collee, J.G., Fraser, A.G., Marmion, B.P., Simmons, A. 2008. Chapter 7: Test for Identification of Bacteria. In: Collee, J.G., Miles, R. S., Watt, B. Eds.Test for Identification of Bacteria, Mackie \& McCartney's Practical Medical Microbiology, 14th Edition, Indian
Reprint, Churchil Livingstone, 2008; 131-149.

Desai, P.J., Pandit, D., Mathur, M., Gogate, A. 2001. The prevalence, identification and the distribution of various species of Enterococci which were isolated from clinical samples, with special reference to the urinary tract infections in catheterized patients. India J. Med. Microbial., 19: 132- 37.

Fernandes, S.C., Dhanashree, B., et al. 2013. Drug Resistance \& Virulence Determinants in Clinical Isolates of Enterococcus Species. Indian J. Med. Res., 137: 981-985.

Forbes, A.B., Sahm, D.F., Weissfeld, A.S. 2007. Chapter 13: Overview of Bacterial Identification Methods and Strategies. In: Bailey \& Scott's Diagnostic Microbiology 12th Ed., Mosby Elsevier International Edition, 216-247.

Gullberg, R.M., Homann, S.R., Phair, J.P. 1989. Enterococcal bacteremia: analysis of 75 episodes. Rev. Infect. Dis., s2: 74-85.

Kumar, S.G., Adithan, C., Harish, B.N., Sujatha, S., Roy, G., Malini, A. 2013. Antimicrobial resistance in India: A review. J. Nat. Sci. boil. Med., 4(2): 286-291.

Maki, D.G., W.A. Agger. 1988. Enterococcal bacteremia: clinical features, the risk of endocarditis, and management. Medicine (Baltimore), 67: 248-269.

Megam, D.W. 1992. Enterococcal endocarditis. Clin. Infect. Dis., 15: 6371.

Mukherjee, K., Bhattacharjee, D., Chakraborti, G., Chatterjee, S. S.Prevalence and Antibiotic Susceptibility Pattern of Enterococcus Species from Various Clinical 
Samples in a Tertiary Care Hospital in Kolkata.

Oberoi, L., et al. 2010. Multidrug Resistant Enterococci in a Rural Tertiary Care Hospital-A Cause of Concern. $J$. Sci., 12: 157-158.

Parameswarappa, J., Basavaraj, V.P., Basavaraj, C.M. 2013. Isolation, identification and antibiogram of enterococci isolated from patients with urinary tract infection. Ann. Afr. Med., 12: $176-81$.
Ross, P.W. 2006. Streptococci and Enterococci. Mackie and Mccartney's Practical Medical Microbiology, $14^{\text {th }}$ edition. Elsievier, 268-269.

Schaberg, D.R., Culver, D.H., Gaynes, R.P. 1991. Major trends in microbial etiology of nosocomial infections. Am. J. Med., 91(suppl 3B): 72S-75S.

Wayne, P.A. 1993. Methods for Dilution Antimicrobial Susceptibility Tests for Bacteria that Grow Aerobically, M7. Clinical and Laboratory Standards Institute (CLSI - formerly NCCLS).

\section{How to cite this article:}

Dillirani, V., and Suresh, R. 2016. A Study on Antibacterial Resistance Patterns on the Enterococcus Species Isolated in a Tertiary Care Hospital in South India. Int.J.Curr.Microbiol.App.Sci. 5(9): 635-639. doi: http://dx.doi.org/10.20546/ijcmas.2016.509.072 